\title{
AUTHOR INDEX \\ Volume 14
}

Abbas, A., see Paria

Abdalla, E., see Wang

Abdesselam, B., Chakrabarti, A., Chakrabarti, R. \& Segar, J., Maps and twists relating $\mathrm{U}(\operatorname{sl}(2))$ and the nonstandard $\mathrm{U}_{h}(\mathrm{sl}(2))$ : Unified construction

Abe, H., Hashida, J., Muta, T. \& Purwanto, A., Path-integral formulation of Casimir effects in supersymmetric quantum electrodynamics

Adam, C., Theta vacuum in different gauges

Adler, R.J. \& Santiago, D.I., On gravity and the uncertainty principle

Aghamohammadi, A.,

Alimohammadi, M. \&

Khorrami, M., Uniqueness of the minimum of the free energy of the 2-D YangMills theory at large $N$

Agrawal, P., More signatures of the intermediate mass Higgs boson

Ahn, C., Oh, K. \& Tatar, R., The large- $N$ limit of $N=1$ field theories from $\mathrm{F}$ theory

Akeroyd, A.G., Arhrib, A. \& Capdequi Peyranère, M., CPodd Higgs boson production in association with neutral gauge boson in high energy $e^{+} e^{-}$collisions

Albeverio, S. \& Fei, S.-M., Symplectic geometry on quantum plane

Aldaya, V., see Guerrero

Alimohammadi, M. \& Shariati, A., Neutrino oscillation in a space-time with torsion

Alimohammadi, M., see Aghamohammadi

Alkalaev, K.B. \& Lyakhovich, S.L., On the consistency problem of interactions of $(2+1)$ massive spinning particle

Al-Naghmoush, A., A1Thoyaib, S.S. \& Taha, M.O.,
A14(1999)2003
A14(1999)1329

A14(1999)765

A14(1999)1033

A14(1999)185

A14(1999)1371

A14(1999)751

A14(1999)1479

A14(1999)369

A14(1999)2093

A14(1999)549

A14(1999)1689

A14(1999)267

A14(1999)751

A14(1999)2727
The Higgs mass from a positivity condition at finite temperature

Alonso, J.L., Cortés, J.L., Boucaud, P., Carmona, J.M., Polonyi, J. \& van der Sjis, A., Mass protection via translational invariance

Al-Rawaf, A.S., Cosmological consequences of a model with a generalized cosmological constant

Al-Solamy, F. \& Beggs, E.J., Soliton decay, time delays and breathers in the chiral equation

Al-Thoyaib, S.S., see A1Naghmoush

Alves, M., Black hole solutions from two-dimensional Bohmian quantum mechanics

Alves, M., Bazeia, D. \& Bezerra, V.B., Black hole formation in bidimensional dilaton gravity coupled to scalar matter systems

Ameduri, M., Efthimiou, C.J. \& Gerganov, B., On the integrability of the BukhvostovLipatov model

Anchordoqui, L.A., Torres, D.F., Romero, G.E. \& Andruchow, I., In search for natural wormholes

Anchordoqui, L.A., Bergliaffa, S.E.P., Trobo, M.L. \& Birman, G.S., Cylindrically symmetric spinning BransDicke space-times with closed timelike curves

Andreev, I.V., Influence of the source evolution on particle correlations

Andruchow, I., see Anchordoqui

Antoniou, I., Gunzig, E., Nardone, P. \& Pronko, G.P., A Hamiltonian formalism for classical systems interacting with quantum systems
A14(1999)1105

A14(1999)2629

A14(1999)2277

A14(1999)1157

A14(1999)631

A14(1999)2629

A14(1999)2187

A14(1999)2687

A14(1999)2341

A14(1999)791

A14(1999)459

A14(1999)791

A14(1999)2287 
Antonov, D., Ensemble of vortex loops in the Abelian-projected SU(3)-gluodynamics

Apikyan, S.A. \& Sahakyan, D.A., Superconformal field theory with boundary: Spin model

Arhrib, A., see Akeroyd

Arias, P.J. \& Khoudeir, A., Massive gauge axion fields

Avila, M.A., Light quark orbital radius of a heavy quark-light quark system in an $S$-state

Avila, M.A., Orbital radius of a hydrogen-like meson in $S$-, $P$ - and $D$-states

Bagchi, B., Ganguly, A., Bhaumik, D. \& Mitra, A., Higher derivative super-symmetry, a modified CrumDarboux transformation and coherent state

Bal, S. \& Sathiapalan, B., High temperature limit of the $N=$ 2 matrix model

Baleanu, D. \& Karasu, A. (Kalkanli), Lax tensors, killing tensors and geometric duality

Bandos, I. \& Lukierski, J., Tensorial central charges and new superparticle models with fundamental spinor coordinates

Banerjee, R. \& Marino, E.C., A new approach for bosonization of massive Thirring model in three dimensions

Banerjee, S.N., see Bhattacharya

Barrow, J.D., An entropic cosmological principle

Bartels, J. \& Royon, C., A parametrization of the inclusive diffractive cross-section at HERA

Barvinsky, A.O., Kamenshchik, A.Yu. \& Kiefer, C., Origin of the inflationary universe

Basu, B. \& Bhattacharyya, D.P., Charge pick-up phenomena in $\mathrm{U}+\mathrm{Al}$ interaction

Batalin, I. \& Marnelius, R., Open group transformations

Battistel, O., see Brizola
A14(1999)1829

A14(1999)211

A14(1999)2093

A14(1999)2125

A14(1999)113

A14(1999)2059

A14(1999)27

A14(1999)2753

A14(1999)2587

A14(1999)1257

A14(1999)593

A14(1999)951

A14(1999)1067

A14(1999)1583

A14(1999)1083

A14(1999)105

A14(1999)1643

A14(1999)1509
Bazeia, D., see Alves

A14(1999)2687

Beciu, M. \& Culetu, H., Embedding as a substitute for the Kruskal maximal extension

Beckers, J., Brihaye, Y. \& Debergh, N., Supersymmetry of multi-boson Hamiltonians

Beggs, E.J., see Al-Solamy

Belyaev, A. \& Rosenfeld, R., Constraints on electroweak contact interactions from LEP and Tevatron data

Benatti, F. \& Floreanini, R., Dissipative contributions to $\varepsilon^{\prime} / \varepsilon$

Benayoun, M., Eidelman, S.I., Ivanchenko, V.N. \&

Silagadze, Z.K., Spectroscopy at B-factories using hard photon emission

Bergliaffa, S.E.P., see Anchordoqui

Bezerra, V.B., see Alves

Bhattacharya, A., Banerjee, S.N. \& Chakraborti, B., On some characteristic features of the phase transition in a fractal universe

Bhattacharyya, A., Ghosh, S.K. \& Raha, S., Pion and kaon dissociation in hot quark medium

Bhattacharyya, D.P., see Basu

Bhaumik, D., see Bagchi

Bietenholz, W., Exact supersymmetry on the lattice

Birman, G.S., see Anchordoqui Biswas, S.K. \& Gautam, V.P., FCNC process in $B$ decay and the vector meson dominance

Bombelli, L., see Lombardo

Borges, P.F., Boschi-Filho, H. \& Farina, C., Generalized partition functions, interpolating statistics and higher virial coefficients

Boschi-Filho, H., see Borges

Boucaud, P., see Alonso

Bozhilov, P., $D=10$ chiral tensionless super $p$-branes

Bracken, P., Analysis of a renormalization group equation using the prolongation method
A14(1999)397

A14(1999)1

A14(1999)1149

A14(1999)631

A14(1999)1519

A14(1999)2605

A14(1999)1105

A14(1999)2687

A14(1999)951

A14(1999)621

A14(1999)105

A14(1999)27

A14(1999)51

A14(1999)1105

A14(1999)1701

A14(1999)539

A14(1999)1217

A14(1999)1217

A14(1999)2277

A14(1999)1335

A14(1999)2507 
Brautti, G., Heterodyne detection of gravitational waves emitted from binary pulsars

Brevik, I. \& Haugen, O., On the entropy change for a fluid containing swimming microorganisms

Brihaye, Y. \& Kosinski, P., Kinks stability in two-Higgs models

Brihaye, Y. \& Gabriel, C., Skyrme model on $S_{3}$ and harmonic maps

Brihaye, Y., see Beckers

Brihaye, Y. \& Kosinski, P., Weak-QES extensions of the Calogero model

Brizola, A., Battistel, O., Sampaio, M. \& Nemes, M.C., Implicit regularisation technique: Calculation of the two-loop $\varphi_{4}^{4}$-theory $\beta$-function

Brücher, L. \& Franzkowski, J., Self-energies and vertex corrections with two factorizing loops

Buchbinder, I.L. \& Samsonov, I.B., On holomorphic effective actions of hypermultiplets coupled to external gauge superfields

Bukina, E.N., Octet magnetic moments with null instantons and semibosonized NambuJona-Lasinio model

Burko, L.M., Beyond the horizon to unknown territories: The singularity inside black holes

Cabo, A., Lucio, J.L. \& Villanueva, V.M., Central charges and cohomological properties in phase space

Cai, R.-G. \& Soh, K.-S., Critical behavior in the rotating $D$-branes

Calucci, G., Pair production in a time-dependent magnetic field

Calucci, G., Possible instability of the vacuum in a strong magnetic field

Camacho, A., On a quantum equivalence principle
A14(1999)1733

A14(1999)1713

A14(1999)247

A14(1999)893

A14(1999)1149

A14(1999)2579

A14(1999)1509

A14(1999) 881

A14(1999)2537

A14(1999)2525

A14(1999)1015

A14(1999)1855

A14(1999)1895

A14(1999)1183; 2813(E)

A14(1999)2621

A14(1999)275
Camacho, A., Aharonov-Bohm effect and coordinate transformations

Camacho, A., Flavor-oscillation clocks, continuous quantum measurements and a violation of Einstein equivalence principle

Cao, L., see Zhu

Capdequi Peyranère, M., see Akeroyd

Capdequi-Peyranère, M., Is supersymmetric quantum mechanics compatible with duality?

Capozziello, S. \& Lambiase, G., Neutrino oscillations in Brans-Dicke theory of gravity

Carlip, S. \& Scranton, R., Remarks on the "new redshift interpretation"

Carmona, J.M., see Alonso

Casadio, R. \& Harms, B., Charged dilatonic black holes: String frame versus Einstein frame

A14(1999)1445

A14(1999)2545

A14(1999)2179

A14(1999)2093

A14(1999)2657

A14(1999)2193

A14(1999)71

A14(1999)2277

Castagnino, M.A., see Lombardo

Cea, P., Consoli, M., Cosmai, L. \& Stevenson, P.M., Further lattice evidence for a large rescaling of the Higgs condensate

Chakrabarti, A., see Abdesselam

Chakrabarti, R., see Abdesselam

Chakraborti, B., see Bhattacharya

Chapline, G., On the occurrence of a positive vacuum energy in a quantum model for space-time

Chekhov, L., $A D S_{3} / C F T_{2}$ correspondence at finite temperature

Chekhov, L. \& Palamarchuk, K., Two-logarithm matrix model with an external field Chen, B., Itoyama, H. \& Kihara, H., Non-Abelian Berry phase, Yang-Mills instanton and $\mathrm{USp}(2 k)$ matrix model

Chen, D.-M., see Shen
A14(1999)1673

A14(1999)765

A14(1999)765

A14(1999)951

A14(1999)2169

A14(1999)2157

A14(1999)2229

A14(1999)869

A14(1999)239 
Chen, Z.-B., see Fan

Cheng, T.P., Kochelev, N.I. \& Vento, V., Further comments on a vanishing singlet axial vector charge

Chiapparini, M., see Delfino

Choi, K., Kim, H.B. \& Kim, H.D., Moduli stabilization in heterotic $M$-theory

Colangelo, P., Iorio, L. \& Nardulli, G., Reducing the experimental uncertainty on the CKM angle $\alpha$

Colangelo, P. \& De Fazio, F., Radiative leptonic $B_{c}$ decays

Consoli, M., see Cea

Cooperstock, F.I., Does a dynamical system lose energy by emitting gravitational waves?

Cortés, J.L., see Alonso

Cosmai, L., see Cea

Cotrone, A.L., A $Z_{2} \times Z_{2}$ orientifold with spontaneously broken supersymmetry

Coule, D.H., Varying $c$ cosmology and Planck value constraints

Culetu, H., see Beciu

da Cruz, W., Topological invariants and anyonic propagators

Dadhich, N., On electrogravity duality

Dadhich, N., On gravito-electromagnetic duality in general relativity

Dadhich, N. \& Raychaudhuri, A.K., Oscillating non-singular relativistic spherical model

Dadhich, N., Patel, L.K. \& Tikekar, R., Global monopole as dual-vacuum solution in Kaluza-Klein space-time

Dahia, F. \& Romero, C., Conical space-times: A distribution theory approach

Dai, Y.-B., see Zhu

David, J.R., Anti-de Sitter gravity associated with the supergroup $\mathrm{SU}(1,1 \mid 2) \times \mathrm{SU}(1,1 \mid 2)$

Davis, A. \& Hübsch, T., A fermionic Hodge star operator
A14(1999)2695

A14(1999)205

A14(1999)1615

A14(1999)125

A14(1999)1461

A14(1999)2303

A14(1999)1673

A14(1999)1531

A14(1999)2277

A14(1999)1673

A14(1999)2487

A14(1999)2437

A14(1999)1

A14(1999)1933

A14(1999)337

A14(1999)759

A14(1999)2135

A14(1999)2721

A14(1999)1879

A14(1999)2367

A14(1999)1143

A14(1999)965
De Andrade, M.A. \& Toppan, F., Real structures in Clifford algebras and Majorana conditions in any space-time

De Fazio, F., see Colangelo

De Paola, R.D.M., Rodrigues, R.B. \& Svaiter, N.F., Casimir energy of massless fermions in the slab-bag

de Souza Dutra, A. \& Natividade, C.P., Remarks on fermion-boson equivalence in three dimensions

Debergh, N., see Beckers

Debergh, N. \& Remezo, L., On further representations of the two-body Calogero algebra

Delfino, A., Navarra, F.S., Nielsen, M., Prandini, R.B. \& Chiapparini, M., Alternative linear chiral models for nuclear matter

Deminov, M.M. \& Slavnov, A.A., Gluon- $W$-meson scattering via different renormalization schemes

Demir, D.A., $g g \rightarrow \gamma \overline{f f}$ in the strongly interacting phase of the MSSM

Deo, B.B., $Z^{\prime}$-gauge boson and inflation in the electroweak scale

Deo, B.B. \& Singh, L.P., Quasi supersymmetry within the standard model and Higgs mass

Deriglazov, A.A. \& Gitman, D.M., Classical description of spinning degrees of freedom of relativistic particles by means of commuting spinors

Diakonov, D., Potential energy of Yang-Mills vortices in three and four dimensions

Diakonov, D., Vortex solution in $(2+1)$-dimensional pure Yang-Mills theory at high temperatures

Divakaran, P.P. \& Rajasekaran, G., A new mechanism for neutrino mass

A14(1999)1797

A14(1999)2303

A14(1999)2353

A14(1999)307

A14(1999)1149

A14(1999)2517

A14(1999)1615

A14(1999)993

A14(1999)2471

A14(1999)1365

A14(1999)2447

A14(1999)709

A14(1999)1725

A14(1999)1909

A14(1999)913

Djordjević, G.S., Dragovich, B. \& Nešić, L.J., p-adic and adelic free relativistic particle 
Djouadi, A., Impact of the SUSY decays on the search for the MSSM Higgs bosons at the LHC

Dobrev, V.K., Doebner, H.-D. \& Mrugalla, C., Difference analogues of the free Schrödinger equation

Doebner, H.-D., see Dobrev

Doff, A. \& Pisano, F., Charge quantization in the largest leptoquark-bilepton chiral electroweak scheme

Dolan, L. \& Langham, M., Symmetric subgroups of gauged supergravities and AdS string theory vertex operators

Dragovich, B., see Djordjević

Dray, T., see Manogue

Dray, T., see Manogue

Duan, Y., Fu, L. \& Zhang, H., Topological current of point defects and its bifurcation

Dyson, F., A conservative revolutionary

Efthimiou, C.J., see Ameduri

Eidelman, S.I., see Benayoun

El Kinani, E.H., $q$-derivation, sine algebra and $\mathrm{U}_{t}(\mathrm{sl}(2))$

Elze, H.-T., The functional derivation of master equations

Esposito, S., The action of neutrino ponderomotive force on supernova dynamics

Falcone, D., Quark mass matrices and observable quantities

Fan, H.-Y. \& Lin, J.-X., Dynamical Hamiltonian for preassigned time-evolution of fermionic squeezing

Fan, H.-Y., Zou, H. \& Fan, Y., Generalized Fermi partition function obtained via coherent state representation of the density operator

Fan, H.-Y. \& Chen, Z.-B., Entangled eigenstates and squeezing operator for complex scalar fields

Fan, H.-Y., Sun, Z.-H. \& Zou, H., On the inverse of twomode boson operators ( $a-$ $\left.b^{\dagger}\right)$ and $\left(a^{\dagger}-b\right)$

Fan, Y., see Fan
A14(1999)359

A14(1999)1113

A14(1999)1113

A14(1999)1133

A14(1999)517

A14(1999)317

A14(1999)99

A14(1999)1243

A14(1999)2011

A14(1999)1455

A14(1999)2341

A14(1999)2605

A14(1999)1609

A14(1999)2259

A14(1999)1763

A14(1999)1989

A14(1999)855

A14(1999)2471

A14(1999)2695

A14(1999) 2783

A14(1999)2471
Farakos, K. \& Savvidy, G.K., Vacuum structure of gauge theory on lattice with two parallel plaquettes action

Farina, C., see Borges

Fayyazuddin, $\Lambda_{b} \rightarrow \Lambda+D^{0}$ $\left(\bar{D}^{0}\right)$ decays and CP-violation

Fei, S.-M., see Albeverio

Félix, J., On theoretical studies of $\Lambda^{0}$ polarization

Fernández, F.M. \& Guardiola, R., Quantization condition for highly excited states

Field, J.H., Indications for an anomalous right-handed coupling of the $b$-quark from a model-independent analysis of LEP and SLD data on $Z$ decays

Field, J.H., Z-decays to $b$ quarks and the Higgs boson mass

Finster, F., Smoller, J. \& Yau, S.-T., The coupling of gravity to spin and electromagnetism

Fiore, R., Tiesi, A., Masperi, L. \& Mégevand, A., Effect of strong magnetic field on the first-order electroweak phase transition

Fiziev, P. \& Yazadjiev, S., Solar system experiments and the interpretation of the transposed equi-affine theory of gravity as a theory with a variable Planck "constant"

Floreanini, R., see Benatti

Franklin, J., A simple Dirac wave function for a Coulomb potential with linear confinement

Franzkowski, J., see Brücher

Fu, L., see Duan

Fujii K., Homma, Y. \& Suzuki, T., Submodels of nonlinear Grassmann sigma models in any dimension and conserved currents, exact solutions

Fukuyama, T., see Nishiura

Gabadadze, G. \& Kakushadze, Z., A remark on Witten effect for QCD monopoles in matrix quantum mechanics

Gabriel, C., see Brihaye
A14(1999)1753

A14(1999)1217

A14(1999)63

A14(1999)549

A14(1999)827

A14(1999)1237

A14(1999)1687(E)

A14(1999)1815

A14(1999)1053

A14(1999)407

A14(1999)511

A14(1999)1519

A14(1999)2409

A14(1999)881

A14(1999)2011

A14(1999)919

A14(1999)433; 629(E)

A14(1999)2151

A14(1999)893 
Gaitan, R., On path-dependent state space for the Proca field

Ganguly, A., see Bagchi

Gao, H. \& Ma, B.-Q., Exotic hadrons of minimal pentaquark $(q q q q \bar{q})$ states

Garcia de Andrade, L.C., On time dislocation solution of Einstein field equations

Garcia de Andrade, L.C., Distributional torsion of hybrid defects

Gasperini, M., Looking back in time beyond the big bang

Gautam, V.P., see Maiti

Gautam, V.P., see Biswas

Gavai, R.V., Re-assessing the anomalous $J / \psi$-suppression in the CERN NA50 data

Gerganov, B., see Ameduri

Gershtein, S.S., Kiselev, V.V., Likhoded, A.K. \&

Onishchenko, A.I., Spectroscopy of doubly charmed baryons: $\Xi_{c c}^{+}$and $\Xi_{c c}^{++}$

Geyer, B., Gitman, D.M. \& Lavrov, P.M., A modified scheme of triplectic quantization

Ghezelbash, A.M., On the exceptional gauged WZW theories

Ghosh, S.K., see Bhattacharyya

Giri, A.K., see Mohanta

Giri, A.K., see Mohanta

Gitman, D.M., see Geyer

Gitman, D.M., see Deriglazov

Gogberashvili, M., Four dimensionality in non-compact Kaluza-Klein model

Golshani, M., see Motavali

Govindarajan, T.R., see Suneeta

Grabowski, J., Marmo, G. \& Michor, P.W., Construction of completely integrable systems by Poisson mappings

Guardiola, R., see Fernández

Gubarev, F.V., Polikarpov, M.I. \& Zakharov, V.I., Short strings in the Abelian Higgs model

Guendelman, E.I., Scale invariance, new inflation and decaying $\Lambda$-terms
A14(1999)1383

A14(1999)27

A14(1999)2313

A14(1999)93

A14(1999)1719

A14(1999)1059

A14(1999) 1565

A14(1999)1701

A14(1999) 821

A14(1999)2341

A14(1999)135

A14(1999)661

A14(1999)199

A14(1999)621

A14(1999) 161

A14(1999)1775

A14(1999)661

A14(1999)709

A14(1999)2025

A14(1999)2481

A14(1999)349

A14(1999)2109

A14(1999)1237

A14(1999)2039

A14(1999)1043
Guendelman, E.I., Scale invariance and vacuum energy

A14(1999)1397

Guerrero, J. \& Aldaya, V., A perturbative approach to the relativistic harmonic oscillator and unitarity

Gunzig, E., see Antoniou

Gupta, R.K., see Johal

Hari Dass, N.D. \& Soni, V., Z test for QCD

Harms, B., see Casadio

Haschke, O. \& Rühl, W., The construction of trigonometric invariants for Weyl groups and the derivation of the corresponding exactly solvable Sutherland models

Hashida, J., see Abe

Haugen, O., see Brevik

Homma, Y., see Fujii

Horvat, R., Stringent constraint on the scalar-neutrino coupling constant from quintessential cosmology

Hosotani, Y. \& Nakayama, R., The Hamilton-Jacobi equations for strings and $p$-branes

Huang, C.-S., Huo, W.-J. \& Wu, Y.-L., The $B \rightarrow X_{s} l^{+} l^{-}$and $B \rightarrow X_{s} \gamma$ decays with the fourth generation

Hübsch, T., see Davis

Huo, W.-J., see Huang

Husain, V. \& Jaimungal, S., Phase transition in quantum gravity?

Ikemori, H., Kitakado, S., Otsu, H. \& Sato, T., Hopf map and quantization on sphere

Iorio, L., see Colangelo

Ito, K., Green-Schwarz superstrings on $A d S_{3}$ and the boundary $N=4$ superconformal algebra

Itoyama, H., see Chen

Ivanchenko, V.N., see Benayoun

Ivanov, E., Krivonos, S. \& Toppan, F., $N=4$ Sugawara construction on $\widehat{\operatorname{sl}(2 \mid 1)}, \widehat{\operatorname{sl}(3)}$ and $\mathrm{mKdV}$-type superhierarchies

Jadach, S., see Ward

Jafarizadeh, M.A. \& RezaeiAghdam, A., T-duality in
A14(1999)1689

A14(1999)2287

A14(1999)2211

A14(1999)559

A14(1999)1089

A14(1999)937

A14(1999)1033

A14(1999)1713

A14(1999)919

A14(1999)2245

A14(1999)1983

A14(1999)2453

A14(1999)965

A14(1999)2453

A14(1999)1079

A14(1999)2649

A14(1999)1461

A14(1999)2379

A14(1999)869

A14(1999)2605

A14(1999)2673

A14(1999)491 
sigma models with KaluzaKlein metric as electric-magnetic duality

Jaimungal, S., see Husain

Jain, P. \& Ralston, J.P., Anisotropy in the propagation of radio polarizations from cosmologically distant galaxies

Jellal, A., Realization of supersymmetric sine algebra and quantum superalgebra $\mathrm{U}_{q}(\operatorname{sl}(2 / 1))$

Jin, C.H., Determinations of $\left|V_{u b}\right|$ and $\left|V_{c b}\right|$ from measurements of $B \rightarrow X_{u, c} \ell v$ differential decay rates

Jin, X. \& Malheiro, M., Nucleon sigma term and inmedium quark condensate in the modified quark-meson coupling model

Joglekar, S.D. \& Misra, A., A derivation of the correct treatment of $\frac{1}{(\eta \cdot k)^{p}}$ singularities in axial gauges

Johal, R.S. \& Gupta, R.K., Quantum group $\mathrm{SU}_{q}(1,1)$ for complex $q$-deformation

Jurčišin, M. \& Kazakov, D.I., Infrared quasi fixed points and mass predictions in the MSSM II: Large $\tan \beta$ scenario

Jurčišin, M., see Yeghiyan

Kakushadze, Z., see Gabadadze

Kamath, S.G., Operator regularization, scale and conformal anomalies for the Landau problem

Kamenshchik, A.Yu., see Barvinsky

Karasu, A. (Kalkanli), see Baleanu

Katoch, A.C., see Sharma

Kaul, R.K., see Suneeta

Kazakov, D.I., see Yeghiyan

Kazakov, D.I., see Jurčišin

Kechkin, O.V., Rotating Bonnor solution in dilatonaxion gravity

Kennedy, D.C., CPT- and $B$ violation: The $p-\bar{p}$ sector

Ketov, S.V., A manifestly $N=$ 2 supersymmetric BornInfeld action

\section{A14(1999)1497}

A14(1999)1079

A14(1999)417

A14(1999)2253

A14(1999)1163

A14(1999)289

\section{A14(1999)2083}

A14(1999)2211

A14(1999)671

A14(1999)601

A14(1999)2151

A14(1999)1391

A14(1999)1083

A14(1999)2587

A14(1999)1841

A14(1999)349

A14(1999)601

A14(1999)671

\section{A14(1999)1599}

A14(1999)849

A14(1999)501
Khanna, M.P., see Sinha

Khorrami, M., see Aghamohammadi

Khoudeir, A., see Arias

Kiefer, C., see Barvinsky

Kihara, H., see Chen

Kim, H.B., see Choi

Kim, H.D., see Choi

Kim, N.J., see Myung

Kimura, K. \& Takamura, A., Exact parametrization of Majorana neutrino mass matrix with large mixing

Kimura, T., Self-duality of super D3-brane action on $A d S_{5} \times S^{5}$ background

Kirchbach, M., Three-flavor symmetry of hadrons consistent with the Okubo-ZweigIizuka rule

Kiselev, V.V., see Gershtein

Kitakado, S., see Ikemori

Kleihaus, B., Energy barrier in the two-Higgs model

Klishevich, S. \& Plyushchay, M., Supersymmetry of parafermions

Kochelev, N.I., see Cheng

Koike, M. \& Sato, J., Effects of matter density fluctuation in long baseline neutrino oscillation experiments

Kong, O.C.W., Neutrino oscillations and flavor structure of supersymmetry without $R$ parity

Kosinski, P., see Brihaye

Kosiński, P. \& Maślanka, P., Deformed Galilei symmetry

Kosinski, P., see Brihaye

Krivonos, S., see Ivanov

Kubo, K.-I., see Suzuki

Kühne, R.W., Time-varying fine-structure constant requires cosmological constant

Kuriki, R., Ogushi, S. \& Sugamoto, A., Deformation of Schild string

Kuznetsov, A.V. \& Mikheev, N.V., Neutrino-electron processes in a strong magnetic field and plasma

Kyriakopoulos, E., Petrov type I, stationary, axisymmetric,
A14(1999)651

A14(1999)751

A14(1999)2125

A14(1999)1083

A14(1999) 869

A14(1999) 125

A14(1999) 125

A14(1999)575

A14(1999)1625

A14(1999)327

A14(1999)81; 879(E)

A14(1999)135

A14(1999)2649

A14(1999)1431

A14(1999)2739

A14(1999)205

A14(1999)1297

A14(1999)903

A14(1999)247

A14(1999)2139

A14(1999)2579

A14(1999)2673

A14(1999)1403

A14(1999)1917

A14(1999)1123

A14(1999)2531 
perfect fluid solution of Einstein's equations

A14(1999)7

Lambiase, G., see Capozziello

Lan, D.M., A quantum potential approach for the gravitational Schrödinger equation and its reduction into the Lane-Emden equation of index 2

Landsteiner, K., String corrections to the Hawking-Page phase transition

Langham, M., see Dolan

Laperashvili, L.V. \& Nielsen, H.B., Dirac relation and renormalization group equations for electric and magnetic fine structure constants

Lavrov, P.M., Neto, J.A. \& Oliveira, W., A proposal for a generalized canonical $\operatorname{osp}(1,2)$ quantization of dynamical systems with constraints

Lavrov, P.M., see Geyer

Lee, H.W., see Myung

Lee, J. \& Sin, S.-J., Duality in $\mathrm{SU}(N) \times \mathrm{SU}\left(N^{\prime}\right)$ product group from $\mathrm{M}$ theory

Li, Z.-H., Quantum ergosphere and Hawking process

Liccardo, A., Pezzella, F. \& Marotta, R., Consistent offshell tree string amplitudes

Likhoded, A.K., see Gershtein

Lin, J.-X., see Fan

Lombardo, F.C., Castagnino, M.A. \& Bombelli, L., From classical chaos to decoherence in Robertson-Walker cosmology

Lu, W.F., Crucial dependence of "precarious" and "autonomous" $\phi^{4}$ 's upon the normalordering mass

Lucio, J.L., see Cabo

Lukierski, J., see Bandos

Lyakhovich, S.L., see Alkalaev

Maślanka, P., see Kosiński

Ma, B.-Q., see Gao

Ma, E., Spontaneous supersymmetric generation of an indeterminate mass scale and a possible light sterile neutrino
A14(1999)2193

A14(1999)2667

A14(1999)379

A14(1999)517

A14(1999)2797

A14(1999)569

A14(1999)661

A14(1999) 575

A14(1999)527

A14(1999)1951

A14(1999)799

A14(1999) 135

A14(1999)855

A14(1999)539

A14(1999)1421

A14(1999)1855

A14(1999) 1257

A14(1999)2727

A14(1999)2139

A14(1999)2313

A14(1999)1637
Machado, A.A. \& Pisano, F., Charged right-handed currents in the leptoquarkbilepton flavor dynamics

Magpantay, J.A., The confinement mechanism in YangMills theory?

Maiti, A.K., Routh, H. \& Gautam, V.P., Radiative rare decay of kaons

Malheiro, M., see Jin

Malik, R.P., Topological features in non-Abelian gauge theory

Malkawi, E. \& Yuan, C.-P., Low energy data and a model of flavor mixing

Mangiarotti, L. \&

Sardanashvily, G., The Koszul-Tate cohomology in covariant Hamiltonian formalism

Mano, S., $A d S_{3}$ gravitational instantons from conformal field theory

Manogue, C.A. \& Dray, T., Dimensional reduction

A14(1999)2223

A14(1999)447

A14(1999)1565

A14(1999)289

A14(1999)1937

A14(1999)1487

A14(1999)2201

A14(1999)1961

A14(1999)99

Manogue, C.A. \& Dray, T., Octonionic Möbius transformations

A14(1999)1243

Mansour, M. \& Tahri, E.H., A $q$-deformation of Virasoro and U(1) Kac-Moody algebras with Hopf structure

Manzoni, L.A., Pimentel, B.M. \& Tomazelli, J.L., Axial anomaly through analytic regularization

Mardoyan, L.G., Sissakian, A.N. \& Ter-Antonyan, V.M., Hidden symmetry of the Yang-Coulomb monopole

Marino, E.C., see Banerjee

Marmo, G., see Grabowski

Marnelius, R., see Batalin

Marotta, R., see Liccardo

Marques, G.C. \& Spehler, D., Chiral asymmetry and gauge invariance

Martín, I., Non-Abelian dual maps in path space

Masperi, L., see Fiore

Masuda, T. \& Saito, S., Supersymmetric extension of
A14(1999)809

A14(1999)1303

A14(1999)593

A14(1999)2109

A14(1999)1643

A14(1999)799

A14(1999)1317

A14(1999)407 
Moyal algebra and its application to the matrix model

Matsuda, K., see Nishiura

Matsuo, T. \& Yokono, T., String creation in D6-Brane background

Mégevand, A., see Fiore

Meljanac, S., Milekovic, M. \& Ristic, R., On infinite quon statistics and "ambiguous" statistics

Mersini, L., Field configurations and their instability induced by higher dimensions of space-time: An example

Michor, P.W., see Grabowski

Mikheev, N.V., see Kuznetsov

Milekovic, M., see Meljanac

Mitra, A., see Bagchi

Misra, A., see Joglekar

Miyagi, S., Yang-Mills instantons on seven-dimensional manifold of $G_{2}$ holonomy

Mohanta, R. \& Giri, A.K., $B^{-}$meson decays into $D_{1}^{0}+$ $P^{-}\left(V^{-}\right)$in HQET

Mohanta, R. \& Giri, A.K., Nonleptonic weak decays $\bar{B} \rightarrow D_{2}^{*} P(V)$ in HQET

Motavali, H., Salehi, H. \& Golshani, M., Conformal invariance and wave-particle duality

Mrugalla, C., see Dobrev

Mukherjee, K., Gap equation in scalar field theory at finite temperature

Mustafa, M.G., see Paria

Muta, T., see Abe

Myung, Y.S., Kim, N.J. \& Lee, H.W., 6-D black string as a model of the AdS/CFT correspondence

Nakajima, N., see Suzuki

Nakatsu, T. \& Yokoi, N., Comments on Hamiltonian formalism of AdS/CFT correspondence

Nakayama, R., see Hosotani

Nardone, P., see Antoniou

Nardulli, G., see Colangelo

Natale, A.A., Squeezed gluon vacuum and the global color model of QCD
A14(1999)2215

A14(1999)433; 629(E)

A14(1999)1175

A14(1999)407

A14(1999)2413

A14(1999)2393

A14(1999)2109

A14(1999)2531

A14(1999)2413

A14(1999) 27

A14(1999)2083

A14(1999)2595

A14(1999)161

A14(1999)1775

A14(1999)2481

A14(1999)1113

A14(1999) 257

A14(1999)2003

A14(1999)1033

A14(1999)575

A14(1999)1403

A14(1999) 147

A14(1999)1983

A14(1999)2287

A14(1999)1461

A14(1999)2049
Natividade, C.P., see de Souza Dutra

Navarra, F.S., see Delfino

Nešić, L.J., see Djordjević

Nemes, M.C., see Brizola

Nersessian, A. \& Ramos, E., A geometrical particle model for anyons

Neto, J.A., see Lavrov

Nielsen, H.B., see Laperashvili

Nielsen, M., see Delfino

Nieto, L.M., Rosu, H.C. \& Santander, M., Hydrogen atom as an eigenvalue problem in 3-D spaces of constant curvature and minimal length

Nieves, J.F. \& Pal, P.B., Nonuniversal gravitational couplings of neutrinos in matter

Nishimura, M. \& Tanii, Y., Local symmetries in the $A d S_{7} /$ $\mathrm{CFT}_{6}$ correspondence

Nishiura, H., Matsuda, K. \& Fukuyama, T., Constraints of mixing angles from lepton number violating processes

Nishino, H., Alternative formulation for duality-symmetric 11-dimensional supergravity coupled to super M-5-brane

Nojiri, S., Obregon, O. \& Odintsov, S.D., Unified approach to study quantum properties of primordial black holes, wormholes and of quantum cosmology

Obregon, O., see Nojiri

Odintsov, S.D., see Nojiri

Ogushi, S., see Kuriki

Oh, K., see Ahn

Oh, P. \& Park, M.-I., Symplectic reduction and symmetry algebra in boundary Chern-Simons theory

Oliveira, W., see Lavrov

Onishchenko, A.I., see Gershtein

Otsu, H., see Ikemori

Özer, H.T., Miura-like free field realization of fermionic Casimir $\mathcal{W B}_{3}$ algebras

Padmanabhan, T., Probing the quantum microstructure of space-time

Pal, P.B., see Nieves
(1999) 231

A14(1999)307

A14(1999)1615

A14(1999) 317

A14(1999)1509

A14(1999)2033

A14(1999)569

A14(1999)2797

A14(1999)1615

A14(1999)2463

A14(1999)1199

A14(1999)2709

A14(1999)433; 629(E)

A14(1999)977

A14(1999)1309

A14(1999)1309

A14(1999)1309

A14(1999)1123

A14(1999)369

A14(1999)569

A14(1999)135

A14(1999)2649

A14(1999)469

A14(1999)1667

A14(1999)1199 
Palamarchuk, K., see Chekhov

Palev, T.D., A generalization of the Holstein-Primakoff and the Dyson expansions for the quantum superalgebra $\mathrm{U}_{q}[\mathrm{gl}(n / m)]$

Paria, L., Abbas, A. \& Mustafa, M.G., A gluon plasma giant resonance and colorsingletness at finite temperature

Park, M.-I., see Oh

Pascual-Sánchez, J.-F., Cosmic acceleration: Inhomogeneity versus vacuum energy

Patel, L.K., see Dadhich

Pesando, I., All roads lead to Rome: Supersolvables and supercosets

Pesando, I., On the effective potential of the $D p-\overline{D p}$ system in type II theories

Pesando, I., On the quantization of the GS type IIB superstring action on $A d S_{3}$ with NSNS flux

Pezzella, F., see Liccardo

Pimentel, B.M., see Manzoni

Pimentel, L.O., Inhomogeneous string cosmology solutions with regular space-time curvature

Pisano, F., see Doff

Pisano, F., see Machado

Plyushchay, M., see Klishevich

Pol'shin, S.A., Massless fields over $R^{1} \times H^{3}$ space-time and coherent states for the Lorentz group

Polikarpov, M.I., see Gubarev

Polonyi, J., see Alonso

Prandini, R.B., see Delfino

Pronko, G.P., see Antoniou

Purwanto, A., see Abe

Qin, Y.-P., A simple method of determining the Hubble constant

Raha, S., see Bhattacharyya

Rai Dastidar, K., see Rai Dastidar

Rai Dastidar, T.K., A possible anisotropy in black body radiation viewed through nonuniform gaseous matter
A14(1999)2229

A14(1999)299

A14(1999)2003

A14(1999)231

A14(1999)1539

A14(1999)2721

A14(1999)343

A14(1999)1545

A14(1999)2561

A14(1999) 799

A14(1999) 177

A14(1999)43

A14(1999)1133

A14(1999)2223

A14(1999)2739

A14(1999)2119

A14(1999)2039

A14(1999)2277

A14(1999)1615

A14(1999)2287

A14(1999)1033

A14(1999)1073

A14(1999)621

A14(1999)2557

A14(1999)1193
Rai Dastidar, T.K., Father time. I. Does the cosmic microwave background radiation provide a universal arrow of time?

Rai Dastidar, T.K. \& Rai Dastidar, K., Father time. II. A physical basis behind Feynman's idea of (anti)particles moving backward in time, and an extension of the CPT theorem to include nonlocal gauge fields

Rajasekaran, G., see Divakaran

Ralston, J.P., see Jain

Rama, S.K. \& Sathiapalan, B., On the role of chaos in the AdS/CFT connection

Ramos, E., see Nersessian

Rashkov, R.C., Note on the boundary terms in AdS/CFT correspondence for RaritaSchwinger field

Raychaudhuri, A.K., see Dadhich

Remezo, L., see Debergh

Rezaei-Aghdam, A., see Jafarizadeh

Ristic, R., see Meljanac

Roberts, M.D., The string deviation equation

Rodrigues, R.B., see De Paola

Romero, C., see Dahia

Romero, G.E., see Anchordoqui

Rosado, A., Contribution of a neutrino anapole moment to the muon anomalous magnetic moment

Rosales, J.J., see Tkach

Rosales, J.J., see Tkach

Rosenfeld, R., see Zerwekh

Rosenfeld, R., see Belyaev

Rosu, H.C., see Nieto

Routh, H., see Maiti

Royon, C., see Bartels

Rühl, W., see Haschke

Ryang, S., Free energies and probe actions for near-horizon D-branes and D1 + D5 system

Saa, A., Nonexistence theorems for traversable wormholes

Safvan, C.P., see Unnikrishnan Sahakyan, D.A., see Apikyan
A14(1999)2499

A14(1999)2557

A14(1999)913

A14(1999)417

A14(1999)2635

A14(1999)2033

A14(1999)1783

A14(1999)2135

A14(1999)2517

A14(1999)1497

A14(1999)2413

A14(1999)1739

A14(1999)2353

A14(1999)1879

A14(1999)791

A14(1999)929

A14(1999)169

A14(1999)1209

A14(1999)35

A14(1999)397

A14(1999)2463

A14(1999)1565

A14(1999)1583

A14(1999)937

A14(1999)1573

A14(1999)843

A14(1999)479

A14(1999)211 
Sahni, V., Generating $\Lambda$ from the vacuum

Sahu, S., Cosmic rays propagation in a pseudo-scalar medium

Saito, S., see Masuda

Sakai, K. \& Tsuboi, Z., Thermodynamic Bethe Ansatz equation for $\operatorname{osp}(1 \mid 2)$ integrable spin chain

Sakamura, Y., The Higgs mass bound in the SUSY multiHiggs-doublet model

Saleev, V.A., $\Omega_{c c c}$ production via fragmentation at LHC

Salehi, H., see Motavali

Sampaio, M., see Brizola

Samsonov, I.B., see Buchbinder

Sanjay, On direct and crossed channel asymptotics of fourpoint functions in AdS/CFT correspondence

Santander, M., see Nieto

Santiago, D.I., see Adler

Sardanashvily, G., see Mangiarotti

Sasaki, C.A.G., Sasaki, D.G.G. \& Sorella, S.P., Nonlinear vector SUSY for the threedimensional topological massive Yang-Mills theory

Sasaki, D.G.G., see Sasaki

Sathiapalan, B., see Rama

Sathiapalan, B., see Bal

Sato, J., see Koike

Sato, T., see Ikemori

Savvidy, G.K., see Farakos

Scadron, M.D., Kaon weak decays in chiral theories

Scadron, M.D., Pion interactions in chiral field theories

Scranton, R., see Carlip

Segar, J., see Abdesselam

Selyugin, O.V., Hadron spinflip amplitude and slope of differential cross-sections

Shariati, A., see Alimohammadi Sharma, K.K. \& Katoch, A.C., An isospin analysis of nonfactorizable contributions to hadronic decays of bottom mesons

Shen, Y.-G. \& Chen, D.-M., The quantum corrections to
A14(1999)2269

A14(1999)1287

A14(1999)2215

A14(1999)2427

A14(1999)721

A14(1999)2615

A14(1999)2481

A14(1999)1509

A14(1999)2537

A14(1999)1413

A14(1999)2463

A14(1999)1371

A14(1999)2201

A14(1999)391

A14(1999)391

A14(1999)2635

A14(1999) 2753

A14(1999)1297

A14(1999)2649

A14(1999)1753

A14(1999)1273

A14(1999)1349

A14(1999)71

A14(1999) 765

A14(1999) 223

A14(1999)267

A14(1999)1841 the entropy of rotating U(1)

$\otimes \mathrm{U}(1)$-dilaton black holes

Shimizu, T. \& Watanabe, K., A relativistic description of Gentry's new redshift interpretation

Sidharth, B.G., A note on "quarks or leptons?"

Silagadze, Z.K., Mirror world versus large extra dimensions

Silagadze, Z.K., see Benayoun

Sin, S.-J., see Lee

Singh, L.P., see Deo

Sinha, S. \& Khanna, M.P., Beauty-conserving strangeness-changing two-body hadronic decays of beauty baryons

Sissakian, A.N., see Mardoyan Sitenko, Y.A., Chiral symmetry breaking as a consequence of nontrivial spatial topology

Sivaram, C., A non-anthropic origin for a small cosmological constant

Slavnov, A.A., see Deminov

Smoller, J., see Smoller

Socorro, J., see Tkach

Soh, K.-S., see Cai

Soni, V., see Hari Dass

Sorella, S.P., see Sasaki

Spehler, D., see Marques

Sriramkumar, L., On the response of detectors in classical electromagnetic backgrounds

Srivastava, S.K., Dual nature of Ricci scalar and models of the early universe

Stancu, I., Can the superKamiokande atmospheric data predict the solar neutrino deficit?

Stevenson, P.M., see Cea

$\mathrm{Su}, \mathrm{R} . \mathrm{K}$., see Wang

Sugamoto, A., see Kuriki

Sun, Z.-H., see Fan

Suneeta, V., Kaul, R.K. \& Govindarajan, T.R., BTZ black hole entropy from Ponzano-Regge gravity

Suzuki, K., Nakajima, N., Toki, H. \& Kubo, K.-I., Analyzing powers in inclusive pion pro-
A14(1999)239

A14(1999)779

A14(1999)387

A14(1999)2321

A14(1999)2605

A14(1999)527

A14(1999)2447

A14(1999)651

A14(1999)1303

A14(1999)701

A14(1999)2363

A14(1999)993

A14(1999)1053

A14(1999)1209

A14(1999)1895

A14(1999)559

A14(1999)391

A14(1999)1317

A14(1999)1869

A14(1999)1021

A14(1999)689

A14(1999)1673

A14(1999)1329

A14(1999)1123

A14(1999)2783

A14(1999)349 
duction at high energy and the nucleon spin structure

Suzuki, T., BPS analysis of the charged soliton solutions of D-brane world volume theory from the viewpoint of targetspace supersymmetry

Suzuki, T., see Fujii

Svaiter, N.F., see De Paola

Taha, M.O., see Al-Naghmoush

Tahri, E.H., see Mansour

Takamura, A., see Kimura

Takeuchi, Y., Tazaki, Y., Tsai, S.Y. \& Yamazaki, T., Wave packet approach to the equalenergy/momentum/velocity prescriptions of neutrino oscillation

Tanii, Y., see Nishimura

Tatar, R., see Ahn

Tazaki, Y., see Takeuchi

Ter-Antonyan, V.M., see Mardoyan

Terazawa, H., Possible explanation for the superficially negative electron-neutrinomass-square observed in nuclear $\beta$-decays

Thomassen, J.B., Two-dimensional bosonization from variable shifts in the path integral

Tiesi, A., see Fiore

Tikekar, R., see Dadhich

Tkach, V.I., Rosales, J.J. \& Torres, J., On the relation of the gravitino mass and the GUT parameters

Tkach, V.I., Rosales, J.J. \& Socorro, J., Spontaneous breaking of supersymmetry in cosmological models and supergravity theories

Toki, H., see Suzuki

Tomazelli, J.L., see Manzoni

Toppan, F., see De Andrade

Toppan, F., see Ivanov

Torres, D.F., see Anchordoqui

Torres, D.F., Stellar footprints of a variable $G$

Torres, J., see Tkach

Trobo, M.L., see Anchordoqui

Trodden, M. \& Vachaspati, T., What is the homogeneity of our universe telling us?
A14(1999)1403

A14(1999)15

A14(1999)919

A14(1999)2353

A14(1999)2629

A14(1999) 733

A14(1999)1625

A14(1999)2329

A14(1999) 2709

A14(1999)369

A14(1999)2329

A14(1999)1303

A14(1999)957

A14(1999) 745

A14(1999) 407

A14(1999)2721

A14(1999)169

A14(1999)1209

A14(1999)1403

A14(1999) 177

A14(1999) 1797

A14(1999)2673

A14(1999)791

A14(1999)1007

A14(1999) 169

A14(1999)1105

A14(1999)1661
Tsai, S.Y., see Takeuchi

A14(1999)2329

Tsuboi, Z., see Sakai

Unnikrishnan, C.S. \& Safvan, C.P., Experimental test of a quantum-like theory: Motion of electrons in a uniform magnetic field, in a variable potential well

Vachaspati, T., see Trodden van der Sjis, A., see Alonso

Vento, V., see Cheng

Villanueva, V.M., see Cabo

Voss, G.-A., R\&D progress toward future linear colliders

Wang, B., Su, R.-K. \& Abdalla, E., Can three-dimensional extreme black holes develop from their nonextreme counterparts?

Ward, B.F.L. \& Jadach, S., Dokshitzer-Gribov-LipatovAltarelli-Parisi evolution and the renormalization group improved Yennie-FrautschiSuura theory in QCD

Watanabe, K., see Shimizu

Wolf, S., Computation of the central charge for the leading order of the $N=2$ superYang-Mills effective action

Wu, S., Global anomaly matching in supersymmetric gauge theories

Wu, Y.-L., see Huang

Wu, Z.C., Can black holes be created at the birth of the universe?

Xue, S.-S., Neutrino masses and mixings

Yamazaki, T., see Takeuchi

Yau, S.-T., see Smoller

Yazadjiev, S., see Fiziev

Yeghiyan, G.K., Jurčišin, M. \& Kazakov, D.I., Infrared quasi-fixed points and mass predictions in the MSSM

Yokoi, N., see Nakatsu

Yokono, T., see Matsuo

Yuan, C.-P., see Malkawi

Yuuichirou, S., A configuration of 11-dimensional curved superspace as background for supermembrane

Zakharov, V.I., see Gubarev
A14(1999)479

A14(1999)2427

A14(1999)1661

A14(1999)2277

A14(1999)205

A14(1999)1855

A14(1999)1923

A14(1999)1329

A14(1999)491

A14(1999)779

A14(1999)2789

A14(1999)2073

A14(1999)2453

A14(1999)2403

A14(1999)2701

A14(1999)2329

A14(1999)1053

A14(1999)511

A14(1999)601

A14(1999) 147

A14(1999) 1175

A14(1999)1487

A14(1999)2767

A14(1999)2039 
Zerwekh, A.R. \& Rosenfeld, R., Limits on a strong electroweak sector from $e^{+} e^{-} \rightarrow \gamma \gamma+\mathbb{E}$ at LEP2

Zhang, H., see Duan

Zhang, R.B., Vector coherent states for affine superalgebras and representations of $\widehat{\mathrm{osp}}(1 \mid 2)$

Zhong, Z.Z., The types of axisymmetric exact solutions closely related to $n$-solitons for Yang-Mills-Higgs theory

Zhu, S.-L. \& Dai, Y.-B., The effect of $B \pi$ continuum in the
QCD sum rules for the $\left(0^{+}\right.$, $1^{+}$) heavy meson doublet in

A14(1999)35

A14(1999)2011

Zhu, Z.-H. \& Cao, L., WKB

A14(1999)2419

A14(1999)585 HQET wave functions with the induced gravity theory

Zinn-Justin, J., Renormalization of gauge theories and master equation

A14(1999)2367

A14(1999)2179

A14(1999)1227

Znojil, M., Elementary doublets of bound states of the radial Dirac equation

A14(1999)863 Zou, H., see Fan A14(1999)2471

Zou, H., see Fan 